\title{
Behaviour of Intraocular Gases
}

\author{
P. M. JACOBS, J. M. TWOMEY AND P. K. LEAVER \\ London
}

\begin{abstract}
Summary
The changes in volume of intraocular bubbles of air, sulphur hexafluoride, perfluoropropane and mixtures of these gases, were studied in human eyes, following vitrectomy for treatment of retinal detachment. The implications of these findings, for the provision of optimal internal tamponade in the treatment of retinal detachment, are discussed.
\end{abstract}

Intraocular air injection has been used in retinal surgery for at least 50 years. ${ }^{1}$ In 1973 , Norton $^{2}$ proposed the clinical use of sulphur hexafluoride to provide a longer acting gas bubble and other relatively insoluble gases have subsequently been suggested. ${ }^{3.4 .5}$ The behaviour of these gases has been well studied in animals. ${ }^{6,7,8,9}$ The development of a non-invasive method of estimating intraocular gas volume ${ }^{10}$ has allowed a more detailed study of gas behaviour in human patients.

\section{Materials and Methods}

All patients, treated by the authors between October 1985 and October 1987, who had intraocular gas injection following vitrectomy, as part of a retinal re-attachment procedure, were entered into this study. The preretinal space of each eye was either filled with gas by simultaneous drainage of preretinal and subretinal fluid, or a measured volume of gas was injected into the preretinal space after first withdrawing from it an equivalent volume of fluid. If the intention at surgery was to fill the preretinal space, air, or a mixture of air and sulphur hexafluoride (SF6) or air and perfluoropropane (C3F8), was used.

The gas mixtures were prepared by filling a
$500 \mathrm{ml}$ infusion bag with measured volumes of gases and the bag was compressed to deliver the gases to the eye at a pressure of between 20 and $30 \mathrm{~mm} \mathrm{Hg}$ above atmospheric (giving an intraocular pressure at the completion of the exchange of between 20 and $30 \mathrm{~mm} \mathrm{Hg}$ ).

Because of the tendency for pure SF6 or C3F8 to expand within the eye, ${ }^{6,14}$ these gases were always diluted with air when used to fill the posterior segment during surgery. When pure (100 per cent) SF6 of C3F8 was used, a volume was chosen so that subsequent expansion of the gas did not result in a dangerous elevation of intraocular pressure. The volume to be injected was first measured in a standard plastic disposable, sterile syringe and then injected through the pars plana via a $27 \mathrm{~g}$ needle.

Sterility of the gases was ensured by passing through a micropore filter prior to injection into the eye.

Following surgery, the change in volume of the intraocular gas bubble with time was monitored using A-scan ultrasound, as previously described. ${ }^{10}$ Because subretinal fluid distorts the contour of the gas bubble, patients were excluded from further monitoring if there was any visible subretinal fluid or if the view of the retina was insufficiently

From Moorfields Eye Hospital, High Holborn, London

Correspondence to: Paul Jacobs M.A.,F.R.C.S., Department of Ophthalmology, University Hospital, Queen's Medical Centre, Nottingham NG7 2UH 
clear to determine whether or not the retina was completely attached. We also excluded any patient with a post operative defect in the corneal epithelium, in case contact from the A-scan probe inhibited healing. During the period of the study, detailed records of intraocular gas behaviour were obtained from 88 eyes. Seventy-one eyes had some form of scleral buckling and in 17 eyes gas tamponade alone was used. Distortion of the globe by scleral buckling introduces an error in the estimation of intraocular gas volume. ${ }^{10}$ Only data from the eyes without scleral buckling are presented here and data from all 17 eyes are included.

The purpose of this study was to document the behaviour of different gas mixtures when used clinically. It was not designed to compare the effectiveness of different gases in curing retinal detachment. The type and quantity of gas used in each eye was chosen to provide what was felt to be the most appropriate tamponade for that particular case. For this reason, different numbers of eyes received different gases or mixtures.

\section{Results}

The changes in intraocular gas volume with time, for each eye, are presented in four graphs (Fig 1 to 4 ). Intraocular gas volume was not measured during surgery. In Figures 1 to 3 the volume given at time 0 is the volume of the posterior segment of the eye, estimated by A-scan ultrasound in the same way

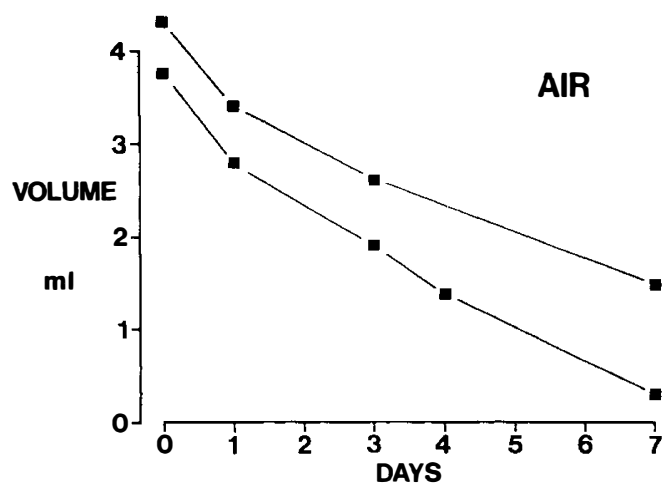

Fig. 1. Change in volume, with time, following surgery, of bubbles of air in two eyes filled with air after vitrectomy. For explanation of volume at time 0 see Fig. 2. as the gas bubble measurements. This is given for reference and assumes a complete fill of gas at the end of surgery. In practice, because of residual subretinal or preretinal fluid, this is seldom achieved and this accounts for the apparent rapid reduction in gas volume in some eyes between day 0 and day 1 . The subsequent volumes for each eye are the actual estimates of intraocular gas volume at a particular time and are not corrected for temperature, atmospheric pressure or intraocular pressure. When a gas volume of 0 is recorded this implies that a tiny bubble was still present, visible ophthalmoscopically, but too small to be detected by the Ascanner.

Figure 1 shows the change in volume with time following surgery for bubbles of air alone, in two phakic eyes, after vitrectomy.

Similar data for the behaviour of 20 per cent SF6 in 7 eyes is given in Figure 2. Except for the large eye, with an initial volume of nearly $16 \mathrm{ml}$, all these eyes were phakic. This group contained a number of myopic and highly myopic eyes with acute retinal detachments. This is reflected in the relatively large initial gas volumes.

Figure 3 charts changes in bubble volume with time for 5 eyes containing mixtures of air and C3F8 in three different initial concen-

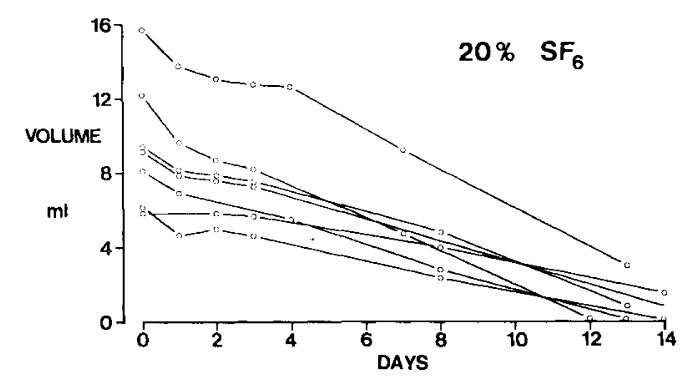

Fig. 2. Change in intraocular gas volume, with time, after surgery for 7 eyes following vitrectomy and filling of the posterior segment with a mixture containing 20 per cent SF6 and 80 per cent air. The volumes given at time 0 were not measured directly but are estimates of the maximum volume of the posterior segment of each eye and are given for reference. In practice, a complete fill of the posterior segment with gas is seldom achieved at surgery and this accounts for the apparent rapid reduction in gas volume for some eyes between day 0 and day 1 . 
trations (16 per cent, 12 per cent and 6 per cent). Only the eye initially containing 16 per cent C3F8 was aphakic.

The intraocular expansions of bubbles of pure gas (100 per cent SF6 and 100 per cent C3F8) are shown in Figure 4.

\section{Discussion}

Gas bubbles have high surface tension and buoyancy in aqueous fluid. ${ }^{11}$ They are, therefore, commonly used after vitrectomy for retinal detachment, both to plug retinal breaks internally and to juxtapose the retina to the underlying retinal pigment epithelium. Parver and Lincoff ${ }^{12}$ showed that a gas bubble of relatively small volume can tamponade a large area of retina. Ideally, tamponade should last at least until a chorioretinal adhesion, from intraoperatively applied laser or cryotherapy, has developed. This probably takes about 12 days. ${ }^{13}$ As indicated in Figure 1 , air does not last long enough to ensure this.

Mixing the less soluble gas SF6 with air approximatley doubles the intraocular duration of the gas (Fig. 2). Twenty per cent SF6 is chosen because it is near the maximum concentration (18.3 per cent) which can be used without causing subsequent expansion of the bubble from influx of nitrogen and oxygen. ${ }^{6}$ Twenty per cent SF6 in air is the agent which, until recently, we used most commonly for internal tamponade following vitrectomy for acute retinal detachment, and it appears to provide adequate tamponade in

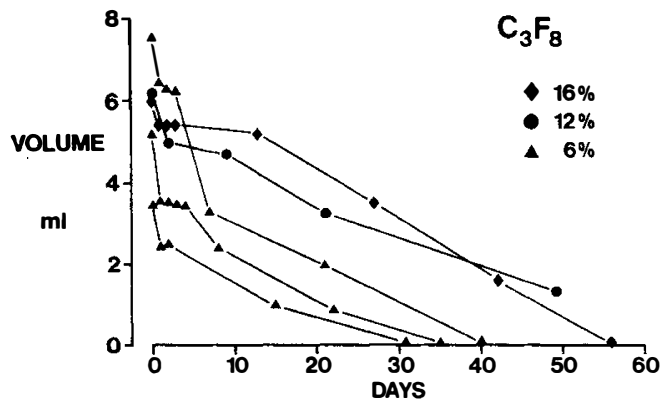

Fig. 3. Change in volume, with time, after surgery, of bubbles of C3F8 mixed with air. The initial proportion of $\mathrm{C} 3 \mathrm{~F} 8$ in the bubble varied as shown. For explanation of volume at time 0 see fig. 2 . most cases. Although a bubble of this mixture persists in an eye for up to two weeks, it begins to shrink immediately and may thus fail to close large retinal breaks or breaks in the inferior part of the retina within two or three days, particularly if the patient has difficulty in adopting an appropriate head posture.

CF8 is very useful in such cases. Our data (Fig. 3) are consistent with those obtained from rabbit eyes ${ }^{14}$ which indicate that the nonexpansile, equilibrated concentration of perfluoropropane gas in the eye is 12 per cent. We have, however, employed 16 per cent $\mathrm{C} 3 \mathrm{~F} 8$ in four eyes (three in conjunction with scleral buckling and therefore not included in Figure 3) without significant postoperative rise in intraocular pressure.

Because C3F8 is so insoluble, bubbles of 12 to 16 per cent C3F8 show very little decline in volume during the first two weeks (Fig. 3) and can thus be used to treat large retinal breaks and breaks in the inferior part of the retina. A disadvantage of such bubbles is that, by persisting in the eye for two months or longer, recovery of useful vision is delayed. As a compromise between 20 per cent SF6 and 12 per cent C3F8 we have found 6 per cent $\mathrm{C} 3 \mathrm{~F} 8$ valuable in some cases. If an eye is filled with 6 per cent C3F8 in air the bubble equilibrates within the first two weeks as nitrogen and oxygen leave the bubble by passing into solution, until the concentration

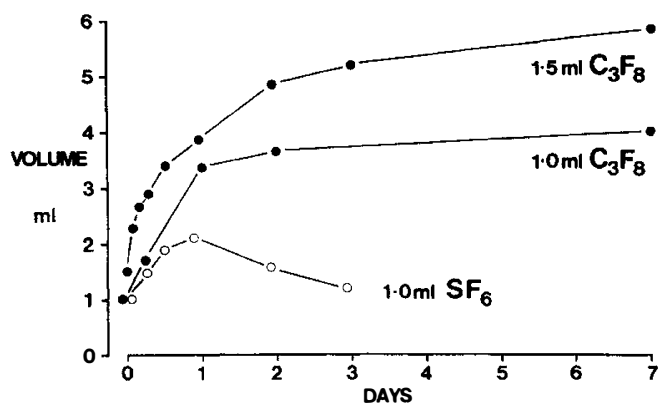

Fig. 4. The rate of expansion of pure SF6 and pure C3F8. Data from 3 different eyes. The volume at time 0 is the volume injected, measured at room temperature and atmospheric pressure. Subsequent measurements are A-scan estimates of intraocular gas volume, uncorrected for atmospheric pressure, intraocular pressure or temperature. 
of C3F8 reaches 12 percent. The bubble is then approximately half its initial volume. Subsequent decline in volume occurs more slowly until disappearance of the bubble takes place at between 30 and 40 days following surgery.

A useful property of SF6 and C3F8 is the capacity for the pure gases to expand by gas transfer. ${ }^{6,9,14}$ As shown in Figure 4, 100 per cent SF6 doubles in volume, while 100 per cent C3F8 will increase to about four times its initial volume. Most of this expansion takes place in the first 24 hours, although some expansion of $\mathrm{C} 3 \mathrm{~F} 8$ continues for four days or longer. By using pure gas, therefore, a large intraocular bubble can be produced without the need to remove subretinal or preretinal fluid. In eyes with normal aqueous outflow the expansion of such bubbles is not associated with significant rise in intraocular pressure. In none of the three eyes documented in Figure 4, did the pressure rise above $20 \mathrm{~mm} \mathrm{Hg}$ at any stage.

Caution must, however, be exercised in attempting a complete fill of an eye using an expanding bubble. If the bubble is still expanding when the posterior segment becomes full, the intraocular pressure rises rapidly and the anterior chamber will shallow. This situation can only be relieved by removal of gas from the posterior segment. When employing large volumes of pure gas (greater than $1.0 \mathrm{ml}$ of $\mathrm{C} 3 \mathrm{~F} 8$ or $2.0 \mathrm{ml}$ of SF6) it is advisable first to estimate the volume of the preretinal space, using ultrasound, to ensure that the expanded bubble will not exceed the space available.

Our evidence shows that air, SF6 and C3F8 behave in a predictable manner in human eyes following surgery. The different properties of these gases allows us to control the volume and duration of the intraocular bubble to produce tamponade appropriate to each case of retinal detachment.

We are grateful for support for this study from the Locally Organised Research Scheme of Moorfields Eye Hospital.

\section{References}

${ }^{1}$ Rosengren B: Cases of retinal detachment treated with diathermy and injection of air into the vitreous body. Acta Ophthalmol 1938, 16: 177 .

2 Norton EWD: Intraocular gas in the management of selected retinal detachments. Trans Am Acad Ophthalmol Otolaryngol 1973, 77: OP85-OP98.

${ }^{3}$ Killey FP, Edelhauser HF, Aaberg TM: Intraocular sulfur hexafluoride and octofluorocyclobutane. Arch Ophthalmol 1978, 96: 511-5.

${ }^{4}$ Lincoff H, Coleman J, Kreissig I, Richard G, Chang S, Wilcox LM: The perfluorocarbon gases in the treatment of retinal detachment. Ophthalmology 1983, 90: 626-41.

${ }^{5}$ Lincoff A, Lincoff $\mathrm{H}$, Iwamoto $\mathrm{T}$, Jacobiec F, Kreissig I: Perfluoro- $\boldsymbol{n}$-Butane. A gas for a maximum duration retinal tamponade. Arch Ophthalmol 1983, 101: 460-2.

6 Abrams GW, Edelhauser HF, Aaberg TM, Hamilton LH: Dynamics of intravitral sulfur hexafluoride gas. Invest Ophthalmol 1974, 13: 863-8.

${ }^{7}$ Lincoff H, Mardirossian J, Lincoff A, Liggett P, Iwamoto $\mathrm{T}$, Jakobiec $\mathrm{F}$ : Intravitreal longevity of three perfluorocarbon gases. Arch Ophthalmol 1980, 98: 1610-11.

${ }^{8}$ Lincoff A, Haft D, Liggett P, Reifer C: Intravitreal expansion of perfluorcarbon bubbles. Arch Ophthalmol 1980, 98: 1646.

${ }^{9}$ Crittenden JJ, de Juan E, Tiedeman J: Expansion of long-acting gas bubbles for intraocular use. Arch Ophthalmol 1985, 103: 831-4.

10 Jacobs PM: Intraocular gas measurement using A-scan ultrasound. Curr Eye Res 1986; 5: 575-8.

11 de Juan E, McCuen B, Tiedeman J: Intraocular tamponade and surface tension. Surv Ophthalmol 1985, 30: 47-51.

12 Parver LM and Lincoff $\mathrm{H}$ : Mechanics of intraocular gas. Invest Ophthalmol 1978, 17: 77-9.

${ }^{13}$ Bloch D, O'Connor P, Lincoff $\mathrm{H}$ : The mechanism of the cryosurgical adhesion III. Statistical analysis. Am J Ophthalmol 1971, 71: 666-73.

14 Peters MA, Abrams GW, Hamilton LH, Burke JM, Schrieber TM: The nonexpansile, equilibrated concentration of perfluorpropane gas in the eye. Am J Ophthalmol 1985, 100: 831-9. 